Estudios de Género de El Colegio de México. 5, 25 de febrero de 2019. e333-e-ISSN.2395-9185

http://dx.doi.org/10.24201/eg.v5i0.333

Artículo

\title{
Círculos virtuosos en la representación política local de mujeres en Chile, 1992-2016
}

\section{Virtuous Cycles in Local Political Representation of Women in Chile, 1992-2016}

Arlette Martínez Ossaำ

Patricio Navia ${ }^{2 *}$

\author{
1 Universidad Diego Portales, Santiago de Chile, email: arlette.martinez@mail.udp.cl \\ ${ }^{2}$ New York University, Nueva York, Estados Unidos, email: patricio.navia@nyu.edu \\ *Autor para correspondencia: Patricio Navia, email: patricio.navia@nyu.edu
}

\section{Resumen}

La presencia de mujeres en cargos de elección popular se asocia a la aparición de círculos virtuosos en la medida que su desempeño ayuda a derribar estereotipos que limitan el acceso de mujeres a dichos cargos. Aquí estudiamos los círculos virtuosos de la presencia de alcaldesas en elecciones en Chile. Con datos de siete elecciones locales - indirectas (1992-2000) y 
directas (2004-2016) — en las 345 municipalidades del país, identificamos que, en el periodo de tres cuatrienios de elecciones indirectas, hubo 59 comunas presididas por una alcaldesa. En el periodo de elecciones directas, esas comunas eligieron más alcaldesas que el resto de las comunas del país. Asociamos que la mayor presencia de mujeres en algunas alcaldías en Chile en la actualidad se debe a la presencia de una alcaldesa en el periodo 19922000.

Palabras claves: Representación de mujeres; representación descriptiva; elecciones locales; alcaldesas; Chile

\begin{abstract}
The presence of women in popularly elected positions is associated to the emergence of virtuous cycles, since their performance in office helps bring down stereotypes that limit the access of women to those positions. We study virtuous cycles in the presence of women in elections in Chile. With data from seven indirect (1992-2000) and direct local elections (2004-2016) in the 345 municipalities of the country, we identify that, in the three fouryear terms of indirect elections, there were 59 municipalities with a woman mayor. In the period with direct elections, those municipalities elected more women than the rest of municipalities in the country. We associate a higher presence of women mayors in Chile today to a higher presence in the 19922000 period.
\end{abstract}

Key words: Gender quotas; women's quotas; representation of women; local elections; mayors; Chile 


\section{Introducción}

La ausencia de mujeres en cargos de elección popular tiende a generar círculos viciosos. En la medida que hay pocas experiencias de mujeres ejerciendo ciertos cargos se seguirán perpetuando los estereotipos de género que dificultan el acceso de otras mujeres a puestos y posiciones similares. Por el contrario, cuando las mujeres logran romper el tejado de vidrio, los estereotipos negativos tienden a desaparecer y resulta más fácil que otras mujeres lleguen a ocupar los mismos cargos u otros similares. Así, la presencia de mujeres en cargos de elección popular funcionaría como una especie de círculo virtuoso en la medida que su desempeño ayuda a derribar barreras que dificultan el acceso de otras mujeres a cargos similares. Con base en lo anterior, debiésemos esperar que en aquellos lugares donde las mujeres han ejercido cargos de representación popular existan más posibilidades de que otras mujeres también ocupen esos cargos. Por el contrario, en lugares donde las mujeres no han tenido — al menos - representación descriptiva, resultará más difícil que alguna mujer acceda a ese tipo de posición.

Aunque en Chile la presencia de mujeres en cargos de elección popular ha aumentado desde el retorno de la democracia en 1990, ésta sigue siendo baja respecto a países occidentales con niveles similares de desarrollo. En los seis periodos de elecciones democráticas locales entre 1992 y 2016, las mujeres han gobernado entre $7.2 \%$ y $12.9 \%$ de los 345 municipios del país. En este artículo estudiamos si aquellas comunas que tuvieron alcaldesas en los primeros tres periodos municipales (1992-2000) —cuando las elecciones eran indirectas - tuvieron más probabilidades de elegir también alcaldesas en el periodo posterior (2004-2016) — de elecciones directas-. Debido a que en los periodos de elecciones indirectas los alcaldes eran escogidos entre los concejales por el propio concejo municipal, los electores de las comunas lideradas por alcaldesas no habían votado necesariamente de manera mayoritaria por una mujer. Sin embargo, las 59 ciudadanas que

llegaron a la alcaldía de forma indirecta pudieron derribar, presumiblemente, estereotipos negativos sobre la capacidad de las mujeres de ejercer cargos en el ámbito municipal. 
Evaluamos si esos 59 municipios tuvieron más probabilidades de elegir una alcaldesa en elecciones directas a partir de 2004.

\section{Los beneficios de la representación descriptiva}

En términos generales, la representación es la autorización que entrega un grupo de individuos a otro para que este último los represente en diversas instancias (Loewenstein, 1965). Para algunos, la representación es concebida como la capacidad de un gobierno para dar respuesta a las preferencias de sus ciudadanos (Dahl, 1971), mientras que para otros es un mecanismo que busca realizar un control entre gobernados y gobernantes (Cotta, 1985). Fuentes y Helgessen (2008) la definen como la delegación en la toma de decisiones de las

altas esferas políticas a través de un proceso de agregación de demandas. Mansbridge (1999) señala que la representación no es un procedimiento pasivo de dar y recibir señales, sino algo dinámico y constitutivo, donde el representado existe en virtud del representante.

Pitkin $(1967,1985)$ identifica cuatro formas de representación: la formal, la simbólica, la sustantiva y la descriptiva. En primer lugar, la representación formal se refiere a las reglas institucionales y procedimientos sobre los que se eligen los representantes; es, por lo tanto, la representación por autorización y responsabilidad. La representación simbólica corresponde a una representación emocional que favorece y explica la aparición de líderes carismáticos, pues reside, en gran medida, en las actitudes y creencias de los representados. La representación sustantiva se refiere a la congruencia entre las acciones de los representantes y los intereses de los representados. En ella se evalúa si los representantes canalizan concreta y efectivamente los intereses de los representados en las distintas dimensiones que son relevantes para los representados. Por último, la autora reconoce otra forma de representación, la descriptiva. Esta ocurre cuando los ciudadanos comparten algunas características con sus representantes. En estas situaciones, decimos que algo es representativo cuando expresamos la idea de similitud, identificación y características compartidas (Sartori, 1992). No obstante, en cualquier caso, la relación entre representantes 
y representados es constitutiva de la democracia como régimen de gobierno y por tanto de su legitimidad y eficacia. En aquellos lugares donde se identifica una debilidad democrática, ésta a menudo está asociada a los vacíos de representación (Ríos, 2008).

Hay buenas razones para creer que la representación descriptiva podría aumentar el sentimiento de confianza política (Ulbig, 2005). Este tipo de representación puede establecer lazos de confianza entre representantes y el electorado, realzando el sentimiento de inclusión y ampliando la confianza tanto en los funcionarios como en las instituciones públicas (Young, 2002; Lawless, 2004; Mansbridge, 1999; Phillips, 1994; Sapiro, 1981; Williams, 2000). Para Dovi (2002), el compromiso con una política de presencia descriptiva es fundamental para apoyar y fortalecer las democracias, pues si se seleccionan representantes descriptivamente se generará una relación mutua con los subgrupos o minorías, se compensará la injusticia de la sub-representación y aumentará la participación política, lo que, a su vez, fortalecerá la legitimidad de las instituciones democráticas. Por su parte, Mansbridge (1999) señala que la representación descriptiva crea un significado social acerca de la capacidad de gobernar respecto de aquellos grupos cuya habilidad ha sido cuestionada. Así, cuando los esfuerzos por mejorar la representación descriptiva se enfocan en cuestiones de género, se revertiría potencialmente la percepción de inferioridad que existe respecto a las mujeres que ocupan posiciones de liderazgo político, generando círculos virtuosos (Alexander, 2012).

Una mujer en el poder permite llevar a la agenda política la perspectiva, problemas e intereses del género (Jones, 2000; Phillips, 1994). Además, aumenta la autoestima de otras mujeres, influye en la autopercepción respecto de su capacidad para asumir roles de liderazgo y en la percepción que tienen los otros respecto a las capacidades de las mujeres. Verba, Burns, y Schlozman (1997) concluyeron que la presencia de mujeres en política incide directamente en la participación de las votantes, pues se incrementa la valoración hacia ellas $\mathrm{y}$, por consiguiente, se contribuye al fortalecimiento de las instituciones democráticas donde participan. A medida que más mujeres son elegidas para un cargo, su rol en la política se vuelve más aceptado, de modo que más mujeres desarrollarán ambiciones políticas, al tiempo que el apoyo entre los votantes también se ampliará (Fox y 
Lawless, 2004; Lawless y Fox, 2010). Todo esto contribuye a romper el tejado de vidrio que dificulta el aumento en el número de las mujeres en cargos de elección popular, ya que se produce un efecto contagio: en la medida que más mujeres ocupen cargos de elección popular, la probabilidad de que otras obtengan ese mismo cargo u otro similar es mayor.

Por el solo hecho de ser mujer, las mujeres pueden compartir características sociales, económicas y políticas con otras mujeres, independientemente de la religión o grupo étnico al que pertenecen (Sapiro, 1981). Dado que plantean prioridades distintas a las que tradicionalmente se han planteado en ambientes en que las mujeres no han estado presentes (Swers, 1998; Thomas y Welch, 1991), las mujeres están mejor posicionadas para representar a otras mujeres (Jones, 2000), independientemente de su ideología. Por lo tanto, los argumentos para apoyar la presencia de mujeres en puestos de representación se basan no solo en representación simbólica o descriptiva, sino también en representación sustantiva.

Si bien un simple aumento en el número de mujeres electas — no una masa crítica - no se traduce necesariamente en un aumento de las políticas para mujeres (Franceschet y Piscopo, 2008), ciertamente a mayor presencia de mujeres habrá también mayores posibilidades de que las distintas formas de representación produzcan resultados más concretos y mejores. De ahí que la ausencia de mujeres en puestos de representación no solo genere debilidades en el sistema democrático y político, sino también falencias a la hora de representar a las mujeres.

\section{Participación política de las mujeres en el ámbito subnacional en América Latina}

La participación política de las mujeres es fundamental no solo para un desarrollo humano sostenible, sino también para mejorar y fortalecer la gobernabilidad democrática (Justiniano, 2013). Se espera que en los órganos de representación haya un número similar 
de hombres y mujeres. No obstante, tanto en el ámbito nacional como en municipal la cantidad de mujeres electas en cargos de representación es considerablemente baja respecto de los hombres.

En las últimas décadas, la política subnacional ha cobrado relevancia en los procesos de expansión de derechos de ciudadanía. Esto se debe en gran medida a la transferencia de responsabilidades de parte del Estado hacia entidades gubernamentales subnacionales (Programa de Naciones Unidas para el Desarrollo, 2013). Se presume que los gobiernos más cercanos, gestionados con base en la participación y rendición de cuentas, están mejor capacitados para desarrollar políticas que faciliten los cimientos para la igualdad de género, por su proximidad espacial y flexibilidad de tiempo para la participación pública (Randall, 1987). Adicionalmente, dan cabida a nuevos actores territoriales que renuevan las redes entre el Estado y la sociedad (Programa de Naciones Unidas para el Desarrollo, 2013).

Por otra parte, los cargos municipales manejan importantes recursos de poder, tienen peso estratégico en la implementación de políticas y constituyen importantes instancias de acumulación de poder político para una proyección nacional (Montero et al., 2004), por ser altamente valorados en la competencia política. Así, aunque las municipalidades son las instancias de gobierno y representación política más próximas a la ciudadanía, además de estar vinculadas a los asuntos de la vida cotidiana, paradójicamente no han facilitado el acceso de las mujeres a los cargos de poder municipal. En esta instancia se produce uno de los déficits más evidentes de las democracias de América Latina. Ríos (2008) destaca que la falta de igualdad en el acceso a cargos públicos y la dificultad para que los intereses de las mujeres sean representados, constituyen problemas evidentes en los regímenes políticos de la región.

En Latinoamérica las mujeres componen el 50\% tanto de la población nacional como del padrón electoral. No obstante, solo representan el 18\% de las autoridades electas en los órganos legislativos de la región (Fuentes y Helgessen, 2008). Asimismo, la cantidad de electas en gobiernos municipales es baja. A pesar de que las cifras se han incrementado con los años, la proporción con respecto a los hombres sigue siendo menor. El informe del 
Programa de Naciones Unidas para el Desarrollo (2013) acerca de la participación política de las mujeres en el ámbito sub-nacional evidencia un crecimiento lento en cuanto a los cargos ejecutivos. Si bien, actualmente hay más mujeres que hace 10 años, el incremento no es significativo. En 1998 el promedio de titulares mujeres era de 5\% y, en 2012, de $11 \%$. En aquel año, de 13,315 cargos de jefatura municipal, solo 1,308 fueron ocupados por mujeres; esto es, un promedio regional de $11 \%$. Ha sido necesaria más de una década para duplicar la presencia de mujeres. En caso de mantenerse este ritmo, tres décadas tardaría en lograrse una masa crítica de mujeres titulares en cargos de representación popular.

El acceso de mujeres a jefaturas municipales es especialmente dificultoso, razón por la que muchos países de la región como Brasil, Costa Rica, Ecuador y Perú han tomado acciones afirmativas bajo el principio de paridad de género. En el caso de los tres primeros, la ley de cuotas municipal precedió a la nacional, mientras que en Perú se establecieron criterios para el emplazamiento de mujeres. Otros países que carecen de ley de cuotas, como Venezuela, han celebrado elecciones bajo criterios de paridad de género, dando pie al desarrollo de la igualdad de oportunidades.

Buendia Sánchez (2014) señala que las mujeres han tenido una participación más frecuente en bases sociales y no en las instancias de toma de decisiones, por lo que se ha considerado fundamental promover su participación a fin de lograr una representación substantiva que

promueva la igualdad de género. Por su parte, Martínez y Garrido (2013) concluyen que la presencia de mujeres en asambleas parlamentarias ha reducido la brecha de género en la representación descriptiva debido al efecto contagio de las políticas de cuotas.

\section{Las razones que explican la subrepresentación de mujeres en cargos de elección popular}

La ausencia de mujeres en cargos de elección popular se explica por diversas variables culturales e institucionales. Inglehart y Norris (2003) demuestran que la existencia de un 
modelo masculino, también denominado como actitudes tradicionales de género, es uno de los principales obstáculos para la elección de mujeres en política. Superarlo comprende, entre otras cosas, poner a prueba los cambios culturales que han experimentado las sociedades (Shvedova, 2005; Valdés y Rebolledo, 2015; Wängnerud, 2009). Dicho modelo determina la forma de hacer política e implica, por ejemplo, no solo que las mujeres deban someterse a campañas con gran violencia discursiva y simbólica (Valdés y Olavarría, 1997), sino también que los votantes consideren que los hombres son mejores líderes (Ballington y Matland, 2004). Adicionalmente, interfieren otras variables como la autonominación, la selección y el respaldo de los partidos, la falta de contactos, la ausencia de sistemas desarrollados de educación y capacitación, además de la propia naturaleza de los sistemas electorales. El problema resulta endógeno pues como las mujeres tienen menos posibilidades de competir en política también tendrán menor capital político para ser candidatas rentables (Iversen y Rosenbluth, 2008).

En general, la participación de mujeres está asociada al compromiso de los partidos para promover su inclusión (Larserud y Taphorn, 2007; Matland, 2006). Sin embargo, esta voluntad refleja una relación de endogeneidad, ya que cuando las mujeres tienen más influencia en política se percibe mayor interés en incorporarlas. Como advierte Sapiro (1981), los sistemas políticos serán más proclives a representar a grupos tradicionalmente subrepresentados cuando éstos desarrollen un sentido de sus intereses y manifiesten sus demandas dentro del sistema. Luego, se desprende que solo cuando haya una masa crítica de mujeres que promuevan mejorar la representación del género, los sistemas políticos reaccionarán ante esas demandas.

Por otro lado, no se puede desestimar la importancia de la selección de candidatos como una de las barreras más significativas que debe superar una mujer para competir en política (De Luca, Jones, y Tula, 2002; Freidenberg y Sánchez, 2002; Rahat y Hazan, 2001; Siavelis, 2002), ya que la influencia que ejercen los órganos directivos es uno de los primeros obstáculos institucionales (Matland, 2002). Para ser candidatas, las mujeres deben enfrentar autoridades partidistas que determinen si serán seleccionadas (Lois y Diz, 2006). Si bien cada colectividad utiliza diversos criterios de selección, los órganos directivos 
influyen directamente en quién será nominado. La nominación puede estar a cargo de una persona, de toda la organización o de los potenciales votantes (Otero, 2008). Por lo tanto, no solo es necesario que haya mujeres que aspiren a ser candidatas, sino también que sean capaces de enfrentar a autoridades partidistas que tienen la capacidad de determinar si serán seleccionadas (Lois y Diz, 2006). En varios países latinoamericanos los partidos poseen el monopolio de las candidaturas y la capacidad exclusiva de nominación (Zovatto, 2007). Dado que el éxito electoral de los partidos dependerá de la nominación (Otero, 2008), las élites políticas tienen mayor interés y capacidades para motivar las candidaturas con pronósticos exitosos (Hinojosa, 2012). Así, la selección conlleva una serie de evaluaciones previas (Freidenberg, 2003), por lo que un partido rechazará a aquellos que parezcan no rentables (Matland, 2002). En contextos institucionales y culturales desfavorables para las mujeres, las potenciales candidatas serán concebidas como no rentables, y lograrán ser nominadas, en el mejor de los escenarios, en lugares donde no tiene posibilidades de triunfar.

\section{El efecto contagio}

Por el contrario, producto del efecto contagio, mientras más candidatas logran la victoria, los partidos se interesarán en promover la participación femenina (Sawer, 2010) y debieran tener más incentivos para nominar mujeres en lugares donde la participación electoral de mujeres es alta. Hay evidencia de la India que fortalece la teoría del efecto contagio; allí se demuestra que es más probable que haya mujeres electas en distritos donde los escaños estaban anteriormente reservados para mujeres (Beaman, Chattopadhyay, Duflo, Pande y Topalova, 2009; Bhavnani, 2009). Asimismo, Tras el estudio de elecciones locales en Estados Unidos, Bhalotra, Clots-Figueras y Iyer (2013) reportaron resultados similares, incluso cuando no existen cuotas de género. Por su parte, Gilardi (2015) señala que también hay efectos positivos en la elección de mujeres en municipios colindantes o cercanos a aquellos donde, en elecciones anteriores, hubiese triunfado una mujer. Para el caso de

Chile, Martínez Ossa y Navia (2017) también reportan más presencia de candidatas en 
lugares donde antes hubo alcaldesas, incluso cuando se excluye a aquellas mujeres que buscaban la reelección. Sin embargo, otros estudios han concluido que el hecho que una mujer consiga un escaño no tiene efectos sobre la probabilidad de que haya más candidatas (Broockman, 2013; Ferreira y Gyourko, 2014).

Se ha identificado que si bien las candidaturas exitosas de mujeres cambian la percepción respecto de la idoneidad de otras mujeres para efectuar exitosamente una carrera política (Lawless y Fox, 2010) y, por consiguiente, lograr que los votantes confíen en las mujeres como representantes (Kanthak y Woon, 2013), estos efectos pueden debilitarse a medida que la presencia de mujeres se vuelve permanente. Particularmente, Gilardi (2015) identifica que la permeabilidad de las mujeres en las elecciones se debe al aumento de nuevas candidatas y no al esfuerzo de hacerlas más elegibles. Por lo tanto, los role models juegan un papel más relevante cuando hay grupos que recientemente se están estableciendo en la arena política (minorías étnicas, personas con discapacidad, homosexuales) y que, aunque han permitido un avance en la representación política de las mujeres, solo ha sido en sus etapas iniciales (Gilardi, 2015). Luego, el efecto de la representación descriptiva puede diluirse en el tiempo en la medida que la mayor presencia de mujeres permite que éstas sean evaluadas a partir de la representación sustantiva (Franceschet y Piscopo, 2008).

Con todo lo anterior, resulta clave identificar si existe una lógica de círculo virtuoso en la forma en que evoluciona la presencia de mujeres en cargos de representación. Si bien hay múltiples razones que explican la subrepresentación de mujeres en cargos de elección popular, es esencial saber si, en lugares donde antes hubo una mujer, aumenta la probabilidad de que otra resulte electa. De existir este patrón, las políticas públicas y el diseño institucional a favor, por ejemplo, de las cuotas de género temporales se hace también esencial. En lugares donde nunca ha habido una mujer, una cuota de género temporal ayudaría a elegir la primera. Así, una vez que las mujeres hayan roto el tejado de vidrio, la probabilidad de que nuevamente sea elegida una mujer en ese cargo sería mayor.

Por eso, postulamos formalmente una hipótesis para evaluar la existencia de círculos virtuosos en la representación descriptiva de mujeres. Específicamente, planteamos que en 
lugares donde anteriormente las mujeres han ocupado un cargo, es más probable que en el futuro otras mujeres sean electas democráticamente a los mismos cargos o a otros similares.

\section{Metodología e hipótesis}

Para evaluar la validez de la hipótesis utilizamos las elecciones locales en Chile. Recopilamos la información de todos los alcaldes que ejercieron su cargo en las 345 comunas de Chile (335 en 1992) entre 1992 y el cuatrienio que se inició a fines de 2016, considerando tres periodos de elecciones indirectas (1992-2000) y cuatro periodos de elecciones directas (2004-2016). No examinamos los patrones de votación de los chilenos ni sus preferencias partidistas, tampoco el efecto de cuotas de género puesto que no se han implementado en las elecciones locales de Chile. Analizamos el efecto de la presencia de alcaldesas en elecciones indirectas sobre la presencia de alcaldesas en el periodo de elecciones directas. Ya que la aplicamos al caso de Chile, nuestra hipótesis específica de trabajo sostiene que, en aquellas comunas de Chile donde hubo una alcaldesa en el periodo de elecciones indirectas (1992-2004), habría más posibilidades de que resultase electa una mujer a partir de 2004 cuando se establecieron las elecciones directas.

Después de presentar los datos, realizaremos una estimación estadística que nos permite identificar el efecto de la presencia de mujeres en alcaldías en el periodo 1992-2000 sobre la posibilidad de que una mujer ganara una elección directa a alcalde en las elecciones realizadas entre 2004 y 2016. Para el modelo, además, incorporamos variables estadísticas de control con datos sociodemográficos de las comunas. Si bien se ha demostrado que estas variables no tienen incidencia sobre la tasa de presencia de candidatas en elecciones locales ni sobre su posibilidad de elegirse (Martínez Ossa y Navia, 2017), incluimos variables tradicionalmente utilizadas — como la ruralidad, pobreza y desarrollo humano- de las comunas como variables de control en nuestros modelos. 
Nótese que no analizamos las razones que afectan el éxito electoral de las candidatas. Luego, no analizamos ni la militancia partidista de las candidatas, ni el número de candidatas que compiten en cada comuna, los patrones de votación histórico en esas comunas o el efecto que tienen las condiciones sociodemográficas de las comunas sobre el desempeño electoral de las candidatas a alcaldesas. Otros estudios ya han dado cuenta del efecto de esas variables (Martínez Ossa y Navia, 2013). Tampoco incluimos otros elementos del sistema electoral como variables independientes, en tanto que no hubo cambios al sistema electoral para las elecciones locales, salvo que se pasara de elecciones indirectas a elecciones directas (y que es nuestra variable independiente de interés).

La Tabla I muestra los estadísticos descriptivos de nuestra variable de interés —la presencia de una alcaldesa en el periodo 1992-2004- y de las variables de control. Adicionalmente, dicha tabla muestra también los estadísticos descriptivos de nuestra variable dependiente, la presencia de una mujer electa en una contienda electoral directa en el periodo 2004-2016.

Tabla I. Elecciones municipales en Chile, 1992-2016 /

Estadísticos descriptivos de las variables

\begin{tabular}{|c|c|c|c|c|c|}
\hline & $\mathrm{N}$ & Mínimo & Máximo & Media & $\begin{array}{l}\text { Desviación } \\
\text { estándar }\end{array}$ \\
\hline Escolaridad promedio (341) & 346 & 5.57 & 13.63 & 8.3263 & 1.43309 \\
\hline $\begin{array}{l}\text { Índice de Desarrollo Humano } \\
\text { (2003) }\end{array}$ & 341 & .51 & .95 & .6876 & .059 \\
\hline Pobreza (2012) & 346 & .00 & 44.6 & 16.99 & 8.05615 \\
\hline Población Rural (2012) & 345 & .00 & 100.00 & 37.51 & 30.09745 \\
\hline $\begin{array}{l}\text { Dummy presencia alcaldesa en } \\
1992-2000\end{array}$ & 345 & .0 & 1.0 & .171 & .3771 \\
\hline $\begin{array}{l}\text { Dummy presencia alcaldesa en } \\
2000-2016 \text { (VD) }\end{array}$ & 345 & .0 & 1.0 & .252 & .4349 \\
\hline
\end{tabular}

VD (Variable dependiente).

Fuente: elaboración propia con datos del Observatorio-Político Electoral (OBPE-UDP) de la Universidad Diego Portales 


\section{Presencia de mujeres en elecciones locales en Chile}

A partir del final de la dictadura (1973-1990), las mujeres pudieron participar como candidatas en las elecciones legislativas y presidenciales de 1989 y en las elecciones municipales de concejales e, indirectamente, de alcaldes realizadas cada cuatro años, entre 1992 y 2000. Posteriormente, a partir de 2004, se establecieron elecciones directas de alcaldes y concurrentes con las de concejales por periodos de cuatro años. Respecto a éstas últimas, en el periodo de elecciones indirectas, los electores escogían concejales en sistemas de listas abiertas, representación proporcional y boletas únicas. El número de concejales variaba entre seis, ocho y diez, dependiendo del número de electores de cada comuna.

Para escoger a los alcaldes, en 1992, los concejales electos designaban de entre ellos mismos al edil. En varios casos, el concejo decidió dividir el periodo de cuatro años en dos subperiodos de dos años, lo que generó que algunas comunas tuvieran dos alcaldes en un cuatrienio. En 1996, como resultado de una reforma a la ley electoral, el candidato a concejal que obtuviera la primera mayoría relativa en una lista cuya votación fuera de al menos $30 \%$ de la votación total sería electo alcalde directamente. Si esa condición no se cumplía, el candidato a concejal con la primera mayoría en la lista más votada sería electo alcalde. Si esa condición tampoco se cumplía, entonces sería electo alcalde aquel candidato con la primera mayoría en la lista más votada (Bunker y Navia, 2010). En caso contrario, el concejo municipal, por voto mayoritario, nombraba al alcalde por el cuatrienio correspondiente. El hecho que los candidatos más votados resultaran electos alcaldes simplificó el proceso, pero no hizo que la elección de alcaldes fuera directa en tanto la victoria de un candidato no dependía exclusivamente de su propia votación sino de la votación acumulada por todos los candidatos de la lista. Esto significa que, cuando una mujer ganaba la alcaldía, lo hacía también con votos emitidos a favor de candidatos varones de esa misma lista.

En teoría, entre 1992 y 2000, el número de candidatos a alcalde era el mismo que el número de candidatos a concejales, en tanto cada candidato a concejal podía potencialmente ser 
electo alcalde, ya fuese en caso de recibir una alta votación o porque lograra conseguir el apoyo de otros concejales electos. A partir de 2004, se separaron las elecciones de alcaldes y concejales. Así, en las contiendas de 2004, 2008, 2012 y 2016 cada una de las 345 comunas del país escogió a un alcalde o alcaldesa de forma directa a través de un sistema mayoritario. Entre 1992 y 2000 no se podía distinguir entre candidatos que aspiraban a ser concejales y aquellos que aspiraban a ser alcaldes. A partir de 2004, se distingue entre candidatos a alcaldes y candidatos a concejales. Por eso, el número de candidatos disminuyó sustancialmente entre 2000 y 2004, como muestra la Tabla II.

Precisamente ese cambio al sistema electoral motiva nuestro estudio comparativo. Evaluamos si en aquellas comunas donde alguna mujer fue electa alcaldesa indirectamente en el periodo 1992-2000, ha habido más probabilidades de que, a partir de 2004, una mujer habría sido electa alcaldesa de forma directa. El hecho que haya habido una mujer en el cargo más importante de una comuna entre 1992 y 2000 —aunque no haya sido electa directamente - pudiera haber ayudado a romper el tejado de vidrio que dificulta el acceso de mujeres a cargos de elección popular.

La presencia de mujeres en alcaldías ha sido baja en Chile. De hecho, el porcentaje de comunas con alcaldesas nunca ha superado el 13\%. Más aún, en el $82.9 \%$ de las 345 comunas que existen hoy en Chile, desde 1992 en adelante, solo ha habido hombres en la alcaldía. Precisamente porque la presencia de mujeres en este tipo de cargos es un hecho tan inusual, queremos entender en qué tipo de comunas es más probable que sea elegida una alcaldesa.

En las elecciones municipales de 1992, de los 6,498 candidatos a concejales, el 16.7\% eran mujeres y de los 2,082 concejales electos, las mujeres constituyeron solo un 12\%. En estas contiendas, el concejo municipal escogió a una mujer para liderar la alcaldía en 21 municipios. En 1996, el porcentaje de mujeres concejales aumentó a 13.8\%. También, el número de comunas con alcaldesas se amplió a 26 (7.6\% del total). En 2000, las mujeres representaron un $16 \%$ de los concejales electos y el número de alcaldesas llegó a 41 (12\% del total de comunas). En los tres periodos de elecciones indirectas en Chile, entre 1992 y 
2000, cincuenta y nueve comunas fueron lideradas por al menos una mujer en algunos de esos periodos. Así, solo un $17.1 \%$ de las comunas del país tuvo una alcaldesa y, por consiguiente, se derribaron los mitos respecto de la capacidad de mujeres para gobernar. Luego, presumiblemente, para la ciudadanía de las comunas donde en la memoria reciente nunca gobernó una mujer, los estereotipos que existían sobre el desempeño de alcaldesas no podían ser contrastados con experiencias concretas de una mujer al mando del gobierno comunal.

En el periodo de elecciones directas, entre 2004 y 2016, el número de mujeres titulares se mantuvo estable: 42 en 2004, 43 en 2008, 43 en 2012 y 40 en 2016. Si bien el número de comunas con mujeres en el cargo administrativo más importante se ha mantenido relativamente estable $-\mathrm{y}$ de hecho es menor en 2016 que en los cuatrienios inmediatamente anteriores — han variado las comunas gobernadas por mujeres.

Como muestra la Tabla II, tras la reforma electoral que significó la elección directa de alcaldes en 2004, de los 1,243 candidatos, 207 (16.7\%) fueron mujeres. Esto es, en nueve de cada 20 comunas se presentaron candidaturas femeninas. En los periodos entre 20082012 y 2012-2016, el 12.5\% de las comunas fue liderada por una mujer. Después de que el número de candidatas aumentó de 207 a 215 entre la elección de 2004 y la de 2008, para la contienda municipal de 2012 disminuyó sustancialmente, a 170 mujeres. Si bien el número total de candidaturas — de hombres y mujeres — se redujo en un 5.8\% entre 2008 y 2012, la disminución de las candidaturas de mujeres fue de un 21.0\%. Así, aunque la disminución en el número de electas fue marginal, representó un mérito, toda vez que el número de candidatas disminuyó. En la contienda de 2016, el número de candidatas aumentó a 230, pero las candidaturas de mujeres se concentraron en pocas comunas. Por ello, en solo 40 comunas resultó ganadora una mujer.

Hay múltiples razones que explican por qué los partidos nominaron más mujeres o por qué algunas de ellas resultaron electas. Sin embargo, nuestro objetivo no es explicar por qué aumenta o disminuye el número de candidatas y de mujeres electas. Lo que queremos evaluar es el efecto de que una mujer haya sido previamente alcaldesa sobre la probabilidad 
Martínez, A., y Navia, P. (2019). Círculos virtuosos en la representación política local de mujeres en Chile[...] 17

de que otras lo sean en elecciones posteriores. Así, comparamos aquellas comunas donde las mujeres fueron visibilizadas en cargos de alcaldesas con aquellas donde nunca hubo mujeres en el principal cargo de administración local.

Tabla II. Mujeres candidatas y ganadoras en elecciones municipales en Chile, 2004-2012

\begin{tabular}{|c|c|c|c|c|c|c|c|}
\hline Año & $\begin{array}{c}\# \\
\text { Comunas }\end{array}$ & $\begin{array}{c}\# \\
\text { Comunas } \\
\text { con } \\
\text { candidatas }\end{array}$ & $\begin{array}{c}\% \\
\text { Comunas } \\
\text { con } \\
\text { candidatas }\end{array}$ & $\begin{array}{c}\# \\
\text { Candidatos } \\
(\mathbf{H}+\mathbf{M})\end{array}$ & $\begin{array}{c}\# \\
\text { Candidatas }\end{array}$ & $\begin{array}{c}\# \\
\text { Electas }\end{array}$ & $\begin{array}{c}\% \\
\text { Comunas } \\
\text { con } \\
\text { alcaldesas }\end{array}$ \\
\hline $1992 *$ & 335 & 321 & 95.8 & 6,498 & 1,085 & 21 & 6.3 \\
\hline $1996 *$ & 342 & 310 & 90.6 & 16,534 & 2,896 & 26 & 7.6 \\
\hline $2000 *$ & 342 & 316 & 92.4 & 13,730 & 2,506 & 41 & 12.0 \\
\hline 2004 & 345 & 155 & 44.9 & 1,243 & 207 & 42 & 12.2 \\
\hline 2008 & 345 & 175 & 50.7 & 1,230 & 215 & 43 & 12.5 \\
\hline 2012 & 345 & 143 & 41.4 & 1,156 & 170 & 43 & 12.5 \\
\hline 2016 & 345 & 168 & 40.0 & 1,211 & 230 & 40 & 11.6 \\
\hline
\end{tabular}

*Elecciones a concejales. Las alcaldesas fueron electas de forma indirecta, por la votación acumulada de todos los candidatos de sus listas

Fuente: elaboración propia con datos de www.elecciones.gov.cl

La Tabla III muestra el cruce de las comunas que tuvieron alcaldesas en el periodo de elecciones indirectas con aquellas que las tuvieron en el periodo de elecciones directas. De las 59 comunas que tuvieron alcaldesas en el periodo de elecciones indirectas, un $64.4 \%$ tuvo alcaldesas en el periodo posterior. Pero de las 286 comunas que no tuvieron alcaldesas en el periodo de elecciones indirectas, solo el $17.1 \%$ tuvo alcaldesas en el periodo posterior. Luego, la tasa de alcaldesas es superior en las comunas donde gobernó al menos una mujer en el periodo 1992-2000 que en aquellas comunas donde no hubo alcaldesa en ese periodo. 
Tabla III. Presencia de alcaldesas en los periodos 1992-2000 y 2000-2016 en Chile

\begin{tabular}{|c|c|c|c|}
\hline $\begin{array}{c}\text { Alcaldesas en periodo } \\
\text { de elecciones } \\
\text { indirectas (1992- }\end{array}$ & \multicolumn{2}{|c|}{$\begin{array}{l}\text { Alcaldesas en periodo de elecciones directas } \\
\qquad(2004-2016)\end{array}$} & 1992-2016 \\
\hline & No & Sí & Total \\
\hline No & $237(82.9 \%)$ & $49(17.1 \%)$ & $286(100 \%)$ \\
\hline Sí & $21(35.6 \%)$ & $38(64.4 \%)$ & $59(100 \%)$ \\
\hline Total & $258(74.8 \%)$ & $87(25.2 \%)$ & $345(100 \%)$ \\
\hline
\end{tabular}

Fuente: elaboración propia con datos del Observatorio-Político Electoral (OBPE-UDP)

de la Universidad Diego Portales

La Tabla IV presenta gráficamente lo que ocurrió entre 2004 y 2016 en las 345 comunas del país. En 59 comunas hubo una alcaldesa en algún momento entre 1992 y 2004. En seis de ellas $(10.2 \%)$ hubo una alcaldesa en los cuatro periodos de elecciones directas. En otras $32(54.2 \%)$, hubo una mujer en uno, dos o tres periodos de elecciones directas. En las 21 comunas restantes $(35.6 \%)$, ninguna mujer fue electa en el periodo de elecciones directas.

Por su parte, en las otras 286 comunas en que no hubo una alcaldesa en el periodo de elecciones indirectas, solo en cuatro casos (1.4\%) hubo una alcaldesa en los cuatro periodos de elecciones directas. En otras 45 comunas (15.7\%) hubo una alcaldesa en uno, dos o tres periodos. En las 237 comunas restantes $(82.9 \%)$ ninguna mujer ha sido electa alcaldesa.

Graficamos la presencia de mujeres en alcaldías de esta forma para mostrar visualmente el efecto que pudo tener la presencia de una mujer en el periodo 1992-2004 (de elecciones indirectas) sobre la presencia posterior de una mujer en el periodo de elecciones directas, a partir de 2004. 
Tabla IV. Presencia de alcaldesas en las 345 comunas de Chile, 1992-2016



Fuente: elaboración propia con datos del Observatorio-Político Electoral (OBPE-UDP) de la UDP 
Identificamos que existe una relación positiva entre las comunas que tuvieron alcaldesas en el periodo de elecciones indirectas y las comunas que han elegido alcaldesas a partir de 2004. Para confirmar estadísticamente el efecto de la presencia de alcaldesas en el periodo de elecciones indirectas sobre la presencia de mujeres en alcaldías en el periodo de elecciones directas, incluyendo otras variables que pudieron haber afectado la presencia de alcaldesas, presentamos un modelo de regresión logística binaria en la Tabla V.

Además de nuestra variable independiente de interés, y siguiendo a Martínez Ossa y Navia (2017), incorporamos variables de control respecto a las condiciones socio-demográficas de las comunas de Chile. Estas variables son la escolaridad, el Índice de Desarrollo Humano comunal (2003), la tasa de ruralidad y de pobreza en cada comuna. Estos datos los obtuvimos del Sistema Integrado de Información Municipal (SINIM) del Ministerio del Interior de Chile.

Los resultados confirman lo reportado por Martínez Ossa y Navia (2017) respecto a que las condiciones sociodemográficas de las comunas chilenas no explican la presencia de mujeres en las alcaldías. Pero los tres modelos de la Tabla $\mathrm{V}$ también muestran que la presencia de alcaldesas en el periodo 1992-2000 explica la presencia de mujeres en las alcaldías en el periodo de elecciones directas.

Los datos muestran que la presencia de mujeres en puestos de elección popular — como las alcaldías - no se explican por características sociodemográficas de las comunas. No hay más alcaldesas en comunas de mayores ingresos, en las más urbanas o en aquellas con mayor nivel de educación. Luego, la presencia de mujeres en cargos de elección popular no está asociada al tipo de electores en la unidad electoral. Por cierto, otros estudios también han mostrado que tampoco afecta si el distrito o unidad electoral vota mayoritariamente por la izquierda o por la derecha (Martínez Ossa y Navia, 2017). 
Tabla V. Regresión logística binaria sobre la presencia de mujeres en alcaldías en periodo de elecciones directas, 2004-2016

\begin{tabular}{|l|c|c|c|}
\hline & Modelo 1 & Modelo 2 & Modelo 3 \\
\hline Escolaridad (2004) & -.041 & -.345 & - \\
\hline IDH (2003) & $(.268)$ & $(.291)$ & 1.979 \\
\hline Ruralidad (2012) & 3.071 & 1.099 & $(2.279)$ \\
\hline Pobreza (2012) & $(6.021)$ & $(6.737)$ & - \\
\hline Presencia de alcaldesas en 1992-2000 & -.003 & -.010 & \\
\hline & $(.008)$ & $(.008)$ & - \\
\hline Constante & -.041 & -0.040 & $(.023)$ \\
\hline 2-log likelihood & $(.020)$ & $(.023)$ & \\
\hline R2 Cox y Snell & - & $2.254 * *$ & $2.175 * *$ \\
\hline R2 Nagelkerke & & $(.350)$ & -2.968 \\
\hline \% predicho correctamente & 330.101 & 284.782 & 330.188 \\
\hline N & .026 & .162 & .143 \\
\hline
\end{tabular}

** Significativo al 0.05

Fuente: elaboración propia con datos del Observatorio-Político Electoral (OBPE-UDP) de la Universidad Diego Portales y datos del Sistema Integrado de Información Municipal (SINIM)

La variable que mejor explica la presencia actual de mujeres en alcaldías en Chile es el hecho que en el periodo 1992-2004 haya habido una mujer que fue escogida alcaldesa por el concejo comunal. El cambio en el sistema electoral, que pasó de elecciones indirectas a elecciones directas de alcaldes, tuvo efectos indirectos de largo plazo sobre la presencia de mujeres. Después de que cambió el sistema de elección de alcaldes, aquellas comunas que tuvieron alcaldesas en el periodo anterior tuvieron más posibilidades de elegir mujeres al cargo más importante de la comuna en el periodo posterior. La evidencia apunta a la presencia de círculos virtuosos. La experiencia de haber tenido alcaldesas en el pasado 
parece haber afectado los estereotipos de género e hizo más probable que esas comunas volviesen a elegir a una mujer.

Precisamente porque ha resultado tan difícil aumentar el número de mujeres en cargos de representación popular en años recientes, las soluciones que se planteen para contribuir a reducir la subrepresentación de mujeres debieran tomar en cuenta la presencia de círculos virtuosos. La adopción de una ley de cuotas transitoria adoptada para las elecciones legislativas de 2017 — y que se mantendrá vigente hasta las elecciones de 2029— debiera contribuir positivamente a mejorar la presencia de mujeres en el poder legislativo.

Ahora bien, ya que hay evidencia de que la presencia de mujeres produce círculos virtuosos, las consecuencias de la ley de cuota probablemente persistirán más allá del periodo en que la ley permanezca vigente. Siguiendo esta lógica y los resultados que aquí presentamos, es posible justificar la adopción de una ley de cuotas temporal también para las elecciones locales.

\section{Conclusiones}

Una de las razones por las que las mujeres han tenido dificultades para aumentar su presencia en cargos de elección popular son los estereotipos que prevalecen respecto a sus capacidades para desempeñar exitosamente esos cargos. Por eso, cuando hay mujeres en cargos de elección popular, su desempeño ayuda a debilitar esos estereotipos, en cuanto que, al igual que los hombres, las hay quienes desempeñan exitosamente sus cargos, así como quienes no lo hacen. La desaparición de los estereotipos de género facilita el acceso de más mujeres a cargos de elección popular - lo que puede ser entendido como un círculo virtuoso-. Por eso, en aquellos lugares en que ya hubo una mujer en un cargo, la posibilidad de que se elija a otra es mayor que en lugares donde nunca una mujer ocupó ese cargo. 
Para contribuir a este debate y evaluar la existencia de círculos virtuosos en la presencia de mujeres en cargos de elección popular, analizamos siete elecciones subnacionales en las 345 comunas de Chile entre 1992 y 2016. Entre 1992 y 2000, las elecciones de alcaldes eran indirectas, por lo que en las comunas lideradas por alcaldesas los votantes no necesariamente habían votado de forma mayoritaria por una mujer. Sin embargo, a partir de 2004 el cargo de jefe comunal comenzó a ser electo directamente. Evaluamos el efecto de la presencia de alcaldesas en el periodo de elecciones indirectas sobre la presencia de alcaldesas en el periodo de elecciones directas. Con un modelo de regresión logística binaria confirmamos ese efecto y descartamos el efecto de variables sociodemográficas.

De las 59 comunas que tuvieron una alcaldesa en al menos uno de los tres periodos de elecciones indirectas, el $64.4 \%$ volvió a tener alcaldesa bajo elecciones directas, mientras que en las 286 comunas que no tuvieron alcaldesa en el periodo de elecciones indirectas solo el $17.1 \%$ tuvo alcaldesa en el periodo posterior a 2004. La evidencia de Chile confirma la existencia de círculos virtuosos: cuando hay mujeres en cargos de elección popular, se debilitan los estereotipos que dificultan la presencia de mujeres en ese tipo cargos, lo que a su vez contribuye a que más mujeres puedan ser electas.

Al mostrar la presencia de círculos virtuosos en el caso chileno, presentamos evidencia a favor del argumento de que, para promover la presencia de mujeres en cargos de elección popular, las cuotas de género — aun si solo rigen por un periodo limitado de tiempocontribuyen a romper de forma permanente los tejados de vidrio.

\section{Agradecimientos}

Los autores agradecen el financiamiento del Fondo de Ciencia y Tecnología de Chile, Fondecyt Regular 1171051 y del Consejo Nacional de Ciencia y Tecnología de Chile, Conicyt/Fondap/15130009 para llevar a cabo esta investigación. 


\section{Referencias bibliográficas}

Alexander, A. C. (2012). Change in women's descriptive representation and the belief in women's ability to govern: A virtuous cycle. Politics \& Gender, 8(4), 437-464. doi: https://doi.org/10.1017/S1743923X12000487

Ballington, J., y Matland, R. E. (2004). Political parties and special measures: Enhancing women's participation in electoral processes. United Nations Office of the Special Adviser on Gender Issues and Advancement of Women (OSAGI), Expert Group Meeting on “Enhancing Women's Participation in Electoral Processes in PostConflict Countries. Recuperado de http://www.un.org/womenwatch/osagi/meetings/2004/EGMelectoral/EP8-

BallingtonMatland.PDF

Beaman, L., Chattopadhyay, R., Duflo, E., Pande, R., y Topalova, P. (2009). Powerful women: does exposure reduce bias? The Quarterly journal of economics, 124(4), 1497-1540. doi: https://doi.org/10.1162/qjec.2009.124.4.1497

Bhalotra, S., Clots-Figueras, I., e Iyer, L. (2013). Path-Breakers: How Does Women's Political Participation Respond to Electoral Success? IZA Discussion Paper, 7771. Recuperado de http://gap.hks.harvard.edu/path-breakers-how-doeswomen $\%$ E2\%80\%99s-political-participation-respond-electoral-success

Bhavnani, R. R. (2009). Do electoral quotas work after they are withdrawn? Evidence from a natural experiment in India. American Political Science Review, 103(1), 23-35. doi: https://doi.org/10.1017/S0003055409090029

Broockman, D. E. (2013). Black politicians are more intrinsically motivated to advance blacks' interests: A field experiment manipulating political incentives. American Journal of Political Science, 57(3), 521-536. doi: 10.1111/ajps.12018 
Buendia Sánchez, J. Y. (2014). Representación política y liderazgo de mujeres. Un estudio comparado sobre Latinoamérica con especial énfasis en Colombia. (Tesis de licenciatura). Universitat de Barcelona, Barcelona. Recuperado de http://diposit.ub.edu/dspace/handle/2445/57957

Bunker, K., y Navia, P. (2010). Democracia municipal en Chile, 1992-2010. Política y Gobierno, 17(2), 243-278.

Cotta, M. (1985). Representación Política. En N. Bobbio (Dir.), Diccionario de Política (pp. 1426 - 1433). México: Siglo Veintiuno.

Dahl, R. (1971). La Poliarquía. Participación y oposición. Madrid: Tecnos.

De Luca, M., Jones, M. P., y Tula, M. I. (2002). Back rooms or ballot boxes? Candidate nomination in Argentina. Comparative Political Studies, 35(4), 413-436. doi: https://doi.org/10.1177/0010414002035004002

Dovi, S. (2002). Preferable descriptive representatives: will just any woman, black, or Latino do? American Political Science Review, 96(4), 729-744. doi: https://doi.org/10.1017/S0003055402000412

Ferreira, F., y Gyourko, J. (2014). Does gender matter for political leadership? The case of US mayors. Journal of Public Economics, 112, 24-39. doi: https://doi.org/10.1016/j.jpubeco.2014.01.006

Fox, R. L., y Lawless, J. L. (2004). Entering the arena? Gender and the decision to run for office. American Journal of Political Science, 48(2), 264-280. doi: https://doi.org/10.1111/j.0092-5853.2004.00069.x

Franceschet, S., y Piscopo, J. M. (2008). Gender quotas and women's substantive representation: Lessons from Argentina. Politics \& Gender, 4(3), 393-425. doi: 
Martínez, A., y Navia, P. (2019). Círculos virtuosos en la representación política local de mujeres en Chile[...] 26

https://doi.org/10.1017/S1743923X08000342

Freidenberg, F. (2003). Selección de candidatos y democracia interna en los partidos de América Latina. Lima: Asociación Civil Tranparencia.

Freidenberg, F., y Sánchez, F. (2002). ¿Cómo se Elige Un Candidato A Presidente? Reglas y prácticas en los partidos políticos de América Latina. Revista de Estudios Políticos (Nueva Época), 118, 321-361

Fuentes, C., y Helgessen, V. (2008). Prefacio. En M. Ríos Tobar (Ed.), Mujer y Política. El impacto de las cuotas de género en América Latina (pp. 11-12). Santiago: Flacso Chile, IDEA Internacional, Editorial Catalonia Ltda.

Gilardi, F. (2015). The temporary importance of role models for women's political representation. American Journal of Political Science, 59(4), 957-970. doi: https://doi.org/10.5167/uzh-114500

Hinojosa, M. (2012). Selecting women, electing women: Political representation and candidate selection in Latin America. Philadelphia: Temple University Press.

Inglehart, R., y Norris, P. (2003). Rising tide: Gender equality and cultural change around the world. e United States of America: Cambridge University Press.

Iversen, T., y Rosenbluth, F. (2008). Work and power: The connection between female labor force participation and female political representation. Annual Reviewof Political Science, 11, 479-495. doi: https://doi.org/10.1146/annurev.polisci.11.053106.151342

Jones, M. (2000). El sistema de cuotas y la elección de las mujeres en América Latina: El papel fundamental del sistema electoral. En P. Saavedra y C. Castro (Coords.), La democracia paritaria en la construcción europea (pp. 35-47). Madrid: CELEM. 
Justiniano, F. (2013). Prólogo. En Centro Regional para América Latina y el Caribe, Panamá ¿Cuánto hemos avanzado? Un análisis de la participación política de las mujeres en los gobiernos subnacionales en América Latina y el Caribe (pp. 13-14). Panama: PNUD.

Kanthak, K., y Woon, J. (2013). Women Don't Run? Representation, Election Aversion, and Gender Differences in Candidate Entry. Recuperado de https://www.vanderbilt.edu/csdi/miller-stokes/02_MillerStokes_WoonKanthak.pdf

Larserud, S., y Taphorn, R. (2007). Designing for Equality. Stockholm: International Institute for Democracy and Electoral Assistance Recuperado de https://www.idea.int/sites/default/files/publications/designing-for-equality.pdf

Lawless, J. L. (2004). Politics of presence? Congresswomen and symbolic representation. Political research quarterly, 57(1), 81-99. doi: https://doi.org/10.1177/106591290405700107

Lawless, J. L., y Fox, R. L., (2010). It still takes a candidate: Why women don't run for office: Cambridge University Press.

Loewenstein, K. (1965). Political power and the governmental process. Chicago: University of Chicago Press.

Lois, M., y Diz, I. (2006). ¿ Qué sabemos sobre la presencia política de las mujeres y la toma de decisiones? Claves para un marco de análisis. Política, 46, 37-60. Recuperado de https://www.redalyc.org/articulo.oa?id=64504602

Mansbridge, J. (1999). Should blacks represent blacks and women represent women? A contingent "yes". The Journal of Politics, 61, 628-657. doi: https://doi.org/10.2307/2647821 
Martínez, M. A., y Garrido, A. (2013). Representación descriptiva y sustantiva: la doble brecha de género en América Latina. Revista mexicana de sociología, 75(3), 407-438.

Martinez Ossa, A., y Navia, P. (2017). Determinantes de la nominación y éxito de las candidatas a alcaldesa en elecciones municipales en Chile, 2004-2012. Revista SAAP. Publicación de Ciencia Política de la Sociedad Argentina de Análisis Político, 11(1), 35-67.

Matland, R. (2002). Estrategias para ampliar la participación femenina en el Parlamento. El proceso de selección de candidatos legislativos y los sistemas electorales. En M. Méndez, y J. Ballington (Eds.), Mujeres en el Parlamento: más allá de los números (pp. 111-134). Suecia: International Institute for Democracy and Electoral Assistance (International IDEA)

Matland, R. E. (2006). Electoral quotas: Frequency and effectiveness. En D. Dahlerup (Ed.), Women, Quotas and Politics (pp. 293-307). New York: Routledge.

Montero, A., Samuels, D., O’Neill, K. M., Bland, G., Eaton, K., Penfold-Becerra, M., Beer, C., M., Wibbels, E., Haggard, S., yWebb, S. B. (2004). Decentralization and democracy in Latin AmericaIndiana: University of Notre Dame Press

Otero, P. (2008). Selección de candidatos en los partidos panameños: Las primarias y sus impactos en el Partido Revolucionario Democrático. Política y gobierno, 15(2), 271314.

Phillips, A. (1994). Democracy and representation: or, why should it matter who our representatives are? Frauen und Politik/Femmes et politiques. 34,63-76. doi: http://doi.org/10.5169/seals-173047.

Pitkin, H. (1967). The Concept of Representation. Berkeley: University of California Press. 
Pitkin, H. (1985). El concepto de representación. Madrid: Centro de Estudios Constitucionales.

Programa de Naciones Unidas para el Desarrollo. (2013). ¿Cuánto hemos avanzado? Un análisis de la participación política de las mujeres en los gobiernos subnacionales en América Latina y el Caribe Panamá: PNDU.

Rahat, G., y Hazan, R. Y. (2001). Candidate selection methods an analytical framework. Party Politics, 7(3), 297-322. doi: https://doi.org/10.1177/1354068801007003003

Randall, V. (1987). Women and politics: An international perspective. Chicago: The University of Chicago Press.

Ríos, M. (2008). Mujer y Política. El impacto de las cuotas de género en América Latina. Santiago: Flacso-Chile, IDEA Internacional, Editorial Catalonia Ltda.

Sapiro, V. (1981). Research Frontier Essay: When Are Interests Interesting? The Problem of Political Representation of Women. The American Political Science Review, 75(3), 701-716. doi: 10.2307/1960962

Sartori, G. (1992). Elementos de teoría política. Madrid: Alianza Editorial.

Sawer, M. (2010). Premature Obituaries: How Can We Tell If the Women's Movement Is Over? Politics \& Gender, 6(4), 602-609. doi: https://doi.org/10.1017/S1743923X10000383

Shvedova, N. (2005). Obstacles to women's participation in parliament. En Julie Ballington y Azza Karam (eds.) Women in parliament: Beyond numbers. Estocolmo, Suecia: IDEA Internacional, 33-51. 
Martínez, A., y Navia, P. (2019). Círculos virtuosos en la representación política local de mujeres en Chile[...] 30

Siavelis, P. (2002). The hidden logic of candidate selection for Chilean parliamentary elections. Comparative Politics, 34(4), 419-438.

Swers, M. L. (1998). Are women more likely to vote for women's issue bills than their male colleagues? Legislative Studies Quarterly, 23(3), 435-448. doi: 10.2307/440362

Thomas, S., y Welch, S. (1991). The impact of gender on activities and priorities of state legislators. The Western Political Quarterly, 44(2), 445-456. doi: https://doi.org/10.1177/106591299104400212

Ulbig, S. G. (2005). Political realities and political trust: Descriptive representation in municipal government. Annual meeting of the Southwestern Political Science Association. Recuperado de http://citeseerx.ist.psu.edu/viewdoc/download?doi=10.1.1.456.699\&rep=rep1\&type= pdf

Valdés, T., y Olavarría, J. (1997). Masculinidad/es: poder y crisis: Santiago de Chile: Isis Internacional.

Valdés, X., y Rebolledo, L. (2015). Géneros, generaciones y lugares: cambios en el medio rural de Chile Central. Polis. Revista Latinoamericana, 42. Recuperado de http://journals.openedition.org/polis/11459

Verba, S., Burns, N., y Schlozman, K. L. (1997). Knowing and caring about politics: Gender and political engagement. The Journal of Politics, 59(4), 1051-1072.

Wängnerud, L. (2009). Women in Parliaments: Descriptive and Substantive Representation. Annual Review of Political Science, 12, 51-69. doi: https://doi.org/10.1146/annurev.polisci.11.053106.123839

Williams, M. S. (2000). Voice, trust, and memory: Marginalized groups and the failings of liberal representation. New Jersey: Princeton University Press. 
Young, I. (2002). Inclusion and democracy: Oxford: Oxford University Press.

Zovatto, D. (2007). América Latina después del 'rally' electoral 2005-2006: algunas tendencias y datos sobresalientes. Revista Nueva Sociedad, 207. Recuperado de http://nuso.org/media/articles/downloads/3403_1.pdf

\section{Sobre los autores}

Arlette Martínez Ossa es cientista política de la Universidad Diego Portales, investigadora de esa universidad y consultora de la Organización Internacional del Trabajo (OIT). Estudia cuestiones de género, participación política y empoderamiento de las mujeres. En 2017 publicó "Determinantes de la nominación y el éxito de las candidatas a alcaldesa en elecciones municipales en Chile, 2004-2012" en la Revista SAAP (Sociedad Argentina de Análisis Político).

Patricio Navia es profesor titular de ciencia política en la Universidad Diego Portales y Clinical Professor of Liberal Studies en New York University. Se especializa en elecciones, opinión pública y estudios legislativos. En 2018 publicó, junto a Rodrigo Osorio, “Attitudes Toward Democracy and Authoritarianism Before, During and After Military Rule. The case of Chile, 1972-2013”, en Contemporary Politics. 